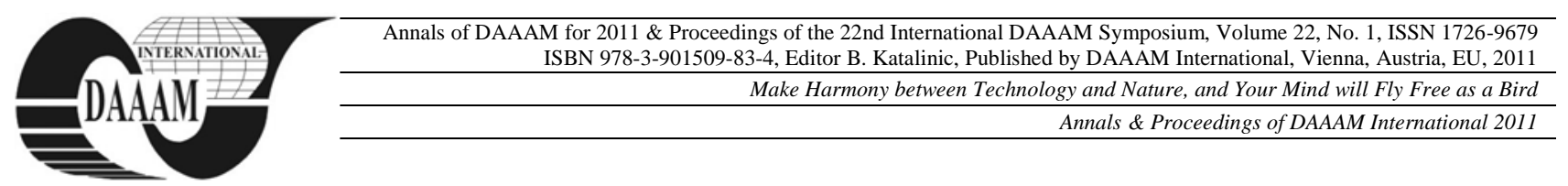

\title{
SOME PROBLEMS AND SOLUTIONS IN NANOROBOT CONTROL
}

\author{
NOVAKOVIC, B[ranko]; MAJETIC, D[ubravko]; KASAC, J[osip] \& BREZAK, D[anko]
}

\begin{abstract}
At the nanoscale the control dynamics is very complex because there are very strong interactions between nanorobots, manipulated objects and nanoenvironment. Therefore the main problems in nanorobotic control are: (i) design of relevant dynamical model of nanorobot motion, (ii) create of the related control algorithm and (iii) measurement at the nanoscale. The main intention in this paper is to highlight the possible ways for solutions of the mentioned problems.
\end{abstract}

Key words: multipotential fields, design of models, measurement in nanorobotics, control in nanorobotics

\section{INTRODUCTION}

The state of the art in the field of nanorobotics has been presented in detail by Novakovic et al., in 2009a. As it is the well known, the nanorobotics is the multidisciplinary field that deals with the controlled manipulation with atomic and molecular-sized objects and therefore sometimes is called molecular robotics (Requicha, 2008). Generally, there are two main approaches for building useful devices from nanoscale components. The first one is based on self-assembly, and is a natural evolution of traditional chemistry and bulk processing (Gómez-López et al., 1996). The second approach is based on control of the positions and velocities of nanoscale objects by direct application of mechanical forces, electromagnetic fields, and the other potential fields. The research in nanorobotics in the second approach has proceeded along two lines. The first one is devoted to the design and computational simulation of robots with nanoscale dimensions (Drexler, 1992). These nanorobots have various mechanical components such as nanogears built primarily with carbon atoms in a diamondoid structure. A big problem is how to build these nanoscale devices.

The second line of nanorobotics research involves manipulation of nanoscale objects with macroscopic instruments and related potential fields. To this approach belong techniques based on Scanning Probe Microscopy (SPM), Scanning Tunneling Microscope (STM, Binnig and Rohrer 1980) and Atomic Force Microscope (AFM, Binning, Quate and Gerber 1986, Stroscio and Eigler 1991). All of these instruments are collectively known as Scanning Probe Microscopes (SPMs). For more information on SPM technology one can see the references (Wiesendanger 1994 and Freitas Jr. 1999). The spatial region in nanorobotics is the bionanorobotics ( Novakovic et al., 2009a and 2009b). Potential applications of the nanorobots are expected in the tree important regions: nanomedicine, nanotechnology and space applications. The complex tasks of the future nanorobots are sensing, thinking, acting and working cooperatively with the other nanorobots.

This paper is organized as follows. The second section presents a design of dynamical model of nanorobot motion in a multipotential field. The third section shows the creation of the related control algorithms. It follows the fourth section where the measurement at the nanoscale has been pointed out. Finally, the conclusion of the paper with some comments and the reference list are presented in the fifth and sixth sections, respectively.

\section{DESIGN OF DYNAMICAL MODEL OF NANOROBOT MOTION}

In order to control nanorobots in mechanics, electronics, electromagnetic, photonics, chemical and biomaterials regions we have to have the ability to construct the related artificial control potential fields. Thus, the first step in designing the dynamics model of nanorobot is the development of the relativistic Hamiltonian $\boldsymbol{H}$ that will include external artificial potential field. This has been done by Novakovic et al,. in 2009a, generally for a multipotential alpha field:

$$
\boldsymbol{H}=\mathrm{E}_{\mathrm{c}}=\mathrm{Hm}_{0} \alpha \alpha^{\prime} \mathrm{c}^{2}+\frac{\mathrm{Hm}_{0} \kappa\left(\alpha-\alpha^{\prime}\right) \mathrm{c} \mathrm{v}}{2} .
$$

Here $\mathrm{v}$ is a nanorobot velocity and $\mathrm{c}$ is the speed of the light both in vacuum without any potential field. Parameters $\alpha$ and $\alpha^{\prime}$ are dimensionless field parameters of a multipotential field in which a nanorobot is propagating and $\kappa$ is an observation parameter. Further, $\mathrm{m}_{0}$ is a nanorobot rest mass and $\mathrm{H}$ is a relativistic parameter. The field parameters $\alpha$ and $\alpha^{\prime}$ can be determined as the dimensionless functions of the total potential energy U. This potential energy includes the all potential energies in the multipotential field that influents to the nanorobot motion, including also the related artificial control potential energy. The notion an alpha field is associated to any potential field that can be described by two dimensionless field parameters $\alpha$ and $\alpha^{\prime}$.

In the nonrelativistic case $(\mathrm{v} r<\mathrm{c})$ and a weak potential field the relation (1) is reduced to the nonrelativistic approximation of the Hamiltonian in an alpha field:

$$
\boldsymbol{H} \cong \mathrm{m}_{0} \mathrm{c}^{2}+\frac{1}{2 \mathrm{~m}_{0}}\left(\mathrm{P}-\frac{\mathrm{v} \mathrm{U}}{\mathrm{c}^{2}}\right)^{2}+\mathrm{U} .
$$

Here $\mathrm{P}=\mathrm{m}_{\mathrm{o}} \mathrm{v}$ is a momentum. In the case where quantum mechanical effects are not present one can employ (2) and classic Hamiltonian canonic forms for designing equations of nanorobot motion in a multipotential field:

$$
\dot{\mathrm{P}}_{\mathrm{i}}=-\frac{\partial \boldsymbol{H}}{\partial \mathrm{q}_{\mathrm{i}}}, \quad \dot{\mathrm{q}}_{\mathrm{i}}=\frac{\partial \boldsymbol{H}}{\partial \mathrm{P}_{\mathrm{i}}} .
$$

In the relation (3) $q_{i}$ and $P_{i}$ are generalized coordinates and momentums, respectively.

In the case where quantum mechanical effects are present for modeling of a nanorobot motion in a multipotential field one should use the following two steps. The first one is to reduce the Hamiltonian from (2) into the kinetic and potential energy only:

$$
\boldsymbol{H} \cong \frac{1}{2 \mathrm{~m}_{0}}\left(\mathrm{P}-\frac{\mathrm{v} \mathrm{U}}{\mathrm{c}^{2}}\right)^{2}+\mathrm{U} .
$$

The second step is to introduce the related Hamiltonian operator: 


$$
\hat{\boldsymbol{H}}=-\frac{\hbar^{2}}{2 \mathrm{~m}_{0}} \nabla_{\mathrm{e}}^{2}+\mathrm{U}(\mathbf{r})
$$

Here $\nabla_{\mathrm{e}}^{2}$ is the extended Laplacian operator, $\hbar$ is the reduced Planck's constant and $\mathbf{r}=(\mathrm{x}, \mathrm{y}, \mathrm{z})$ is the nanorobot position in three-dimensional space. For a general quantum system one can employ time dependent Schrödinger equation (Griffiths, 2004):

$$
\mathrm{i \hbar} \frac{\partial}{\partial \mathrm{t}} \Psi(\mathbf{r}, \mathrm{t})=\hat{\boldsymbol{H}} \Psi(\mathbf{r}, \mathrm{t})
$$

Here $\Psi(\mathbf{r}, t)$ is the wave function, which is the probability amplitude for different configurations of the system. The presented Schrödinger equation describes a particle dynamics without spin effects. For inclusion of the spin effects one should employ the related Dirac's equations (Dirac, 1978).

\section{CREATION OF CONTROL ALGORITHMS}

In the creation of the control algorithms for nanorobot control one should distinguish the two different situations. The first one is the situation where quantum mechanical effects are not present. In that case one can start with the dynamic model of nanorobot motion in a multipotential field (3) and apply any control strategy for control of the nonlinear multivariable dynamical systems. In that sense a very efficient concept of the external linearization in the multipotential field can be applied (Novakovic, 2010):

$$
\mathrm{U}_{\mathrm{c}}=\mathrm{f}\left(\mathrm{U}_{\mathrm{w}}, \mathrm{U}, \mathrm{K}\left(\mathrm{U}_{\mathrm{w}}-\mathrm{U}\right)\right)
$$

In this relation $U_{c}$ is a control potential energy, $U$ is the total potential energy of the nanorobot in the multipotential field, $U_{w}$ is the desired potential energy of the nanorobot in that field and $\mathrm{K}$ is the related controller of the nanorobot motion. Applying the nonlinear control algorithm (7) to the closed loop with the canonical nonlinear differential equations (3) one obtains the linear behavior of the whole system. That is why it is called the external linearization of the nonlinear system. In that case, for design of the controller $\mathrm{K}$, one can use any of the well known procedures for control synthesis of the linear systems (optimal, adaptive and so on).

The second situation is occurred when quantum mechanical effects are present. In that case one can start with the Schrödinger equation (6), or related Dirac's equations (Dirac, 1978) and Dirac's like equations (Novakovic, 2010) and apply the control strategies for control of the quantum mechanical systems. In that sense, dynamics of the quantum feedback systems and control concepts and applications are presented by Yanagisawa and Kimura in 2003.

\section{MEASUREMENT AT THE NANOSCALE}

The main problems in the measurement at the nanoscale are the perturbative effects of the measurement instruments to the nanostructure being investigated. There are several tricks of the trade in atomic force microscopy (AFM) for obtaining images of surface with atomic level resolution. Recently, scientists added a new approach to this toolkit when they showed that terminating an AFM tip in a single carbon monoxide allowed them to image individual atoms in pentacene. This relatively new technique to map out (in three dimensions) the chemical forces between two carbon monoxide molecules has been applied by Sun et al. in 2011. As the oscillating tip of an AFM approaches to the atoms or molecules on a surface, it is experiences both attractive (van der Waals) and repulsive (Pauli) forces. Measuring these forces with sufficient accuracy (one of many applications of AFM) requires that the tip be sufficiently near the surface that these forces exert a sizable shift on its resonance frequency, but not so close that the tip actually bends or moves the molecules. Sun et al. in 2011 identify the optimal distance range within the AFM tip should be moved. A new demonstration of the nonperturbative use of diffraction-limited optics and photon localization microscopy to visualize the controlled nanoscale shifts of zeptoliter mode volumes within plasmonic nanostructures has been presented by McLeod et al. in 2011. Unlike tip or coating based methods for mapping near fields, these measurements do not affect the electromagnetic properties of the structure being investigated.

\section{CONCLUSION}

Some important problems and the related solutions in the region of a nanorobotic control have been pointed out in this paper. For design of the relevant dynamical model of a nanorobot motion we introduced the Hamiltonian for a multipotential field and related canonical equations. In the case where quantum mechanical effects are present this Hamiltonian is transformed into the related Hamiltonian operator and Schrödinger's, or Dirac's equations should be employed. For control of nanorobot motion the external linearization concept has been proposed. Problems and solutions of the measurement at the nanoscale are also discussed in this paper. The further research will be devoted to application of the presented ideas. The limitations of the research and the authors approach are related to the non-quantum systems.

\section{REFERENCES}

Binnig, G.; Quate, C. F. \& Gerber, Ch. (1986). Atomic Force Microscopy. Phys. Rev. Lett. Vol. 56, No. 3 (March 1986), pp. 930-933

Dirac, P.A.M. (1978). Directions in Physics, John Wiley \& Sons, New York

Drexler, K. E. (1992). Nanosystems. John Wiley \& Sons, New York

Freitas, R. A. Jr. (1999). Nanomedicine. Vol. I: Basic Capabilities, Landes Bioscience, Georgetown

Griffiths, D. J. (2004). Introduction to Quantum Mechanics, $2^{\text {nd }}$ edition, Benjamin Cummings, San Francisco

McLeod, A. et al. (2011). Visualization of Nanoscale Plasmonic Nonperturbative Field Distribution via Photon Localization Microscopy, Phis. Rev. Lett., Vol. 106, No. 037402 (January 18, 2011), ISSN 1943-2879

Novakovic, B.; Majetic, D.; Kasac, J. \& Brezak, D. (2009a). Derivation of Hamilton Functions Including Artificial Control Fields in Nanorobotics, Proceedings of $12^{\text {th }}$ International Scientific Conference on Production Engineering - CIM 2009, 17-20th June 2009, Biograd, Croatia, ISBN 953-97181-6-3, Udiljak, T. \& Abele, E. (Ed.), pp. NB 1-8, Published by Croatian Association of Production Engineering, Zagreb

Novakovic, B.; Majetic, D.; Kasac, J. \& Brezak, D. (2009b). Artificial Intelligence and Biorobotics: Is an Artificial Human Being Our Destiny?, Annals of DAAAM for 2009 $\&$ Proceedings of the 20th International DAAAM Symposium, 25-28th November 2009, Vienna, Austria, ISSN 1726-9679, ISBN 978-3-901509-70-4, Katalinic, B. (Ed.), pp. 0121-0122, Published by DAAAM International Vienna, Vienna

Novakovic, B. (2010). Generalized Relativistic Hamiltonian in an Alpha Field, AIP Conference Proceedings 1303, CASYS09, 3-8th August 2009, Liege, Belgium, ISSN 0094-243X, ISBN 978-0-7354-0858-6, Dubois, D. (Ed.), pp. 141-148, AIP-American Institute of Physics, Melville, New York

Sun, Z. et al. (2011). Quantitative Atomic Force Microscopy with Carbon Monoxide Terminated Tips, Phis. Rev. Lett., Vol. 106, No. 046104 (January 27, 2011), ISSN 1943-2879.

Yanagisawa, M. \& Kimura, H. (2003). Transfer Function Approach to Quantum Control, IEEE Trans. On Automatic Control, Vol. 48, No. 12 (December 2003), pp. 2107-2132, ISSN0018-9286 